\title{
Musculoskeletal injuries in the ultramarathon: the 1990 Westfield Sydney to Melbourne run
}

\author{
K E Fallon
}

\begin{abstract}
Objective-To document the injuries sustained by participants in a $1005 \mathrm{~km}$ ultramarathon.

Methods-Clinical notes were reviewed on entrants in the $1005 \mathrm{~km}$ Sydney to Melbourne ultramarathon. An injury was recorded following self referral by a participant or if the history obtained from the runner or his support crew indicated the likelihood of a significant injury which could have an impact upon performance. Results -64 injuries were found in 32 runners. The knee $(31.3 \%)$ and ankle $(28.1 \%)$ regions were most commonly injured. The most common single diagnosis was retropatellar pain syndrome, and Achilles tendinitis and medial tibial stress syndrome were the next most common injuries. Peritendinitis/tendinitis of the tendons passing under the extensor retinaculum at the ankle, an injury infrequently reported in other sports, was common ( $19 \%$ of all injuries).

Conclusions-The injuries were typically associated with running but $12(19 \%$ of the total) involved the tendons of the muscles of the anterior compartment of the lower leg, and in almost every case the major site of inflammation was at the extensor retinaculum at the anterior aspect of the ankle. This injury appears to be relatively specific to the ultramarathon - "ultramarathoner's ankle".

(Br F Sports Med 1996;30:319-323)
\end{abstract}

Key terms: ultramarathon; injuries; tendinitis

Injuries occurring in runners have been well characterised, with several surveys concentrating on presentation of runners to sports medicine clinics. ${ }^{1}$ Less frequent are studies documenting injuries sustained during marathons. ${ }^{23}$

Only two studies have previously addressed musculoskeletal injuries sustained during an ultramarathon running event. Hutson ${ }^{4}$ recorded injuries in participants of a six day track race in which 23 men and two women of average age 41 years ran up to 576 miles. Sixty per cent of participants sustained injuries sufficiently severe to impair their performance. Twenty seven injuries were documented. Minor complaints such as muscle stiffness and blistering of the feet were very common, with the most common specific injuries being tendinitis of the foot dorsiflexors, patellar tendinitis, and achilles tendinitis. These injuries made up $30 \%, 18 \%$, and $18 \%$, respectively, of all injuries.

Rodriguez comments on musculoskeletal findings during two six-day runs held on a 400 metre running track. No specific figures were provided by this investigator, who reported that the most frequently encountered musculoskeletal problems were patellofemoral pain, iliotibial band syndrome, "shin splints", anterior and posterior tibial tendinitis, metatarsal strains, and lower limb oedema. Early compartment syndromes and achilles tendinitis were seen less frequently. ${ }^{5}$

A report regarding causes of withdrawal from the 1988 Sydney to Melbourne ultramarathon indicates that of the 20 withdrawals 12 were related to the musculoskeletal system. The primary diagnoses in these cases were tibialis posterior tendinitis (four cases), stress fracture (two), patellofemoral syndrome (two), quadriceps tears (two), and one case each of achilles tendinitis and ankle sprain. ${ }^{6}$

The only other published report of ultramarathon injuries was a general observation based upon the Western States 100 Mile Run. In this event the common injuries are blisters, muscle damage, and "mechanical strain"; specific mention was made of tendinitis of the anterior ankle.

In view of the relative paucity of information relating to injuries incurred during participation in an ultramarathon running event, an injury analysis of the 1990 Sydney to Melbourne ultramarathon was undertaken.

\section{Methods}

The 1990 Westfield Sydney to Melbourne ultramarathon was a continuous running event of $1005 \mathrm{~km}$. The race was conducted on public roads with runners required for reasons of safety to run only on the left hand side of the road. The running surface varied from smooth bitumen to unsealed road. Environmental conditions varied from $-3^{\circ} \mathrm{C}$ at night during passage across a mountainous region to $27^{\circ} \mathrm{C}$ in the early afternoon of the second day of the run.

The winner completed the event in 5 days, 23 hours and 55 minutes. Of the 32 starters 19 completed the event within the cut off time of 8.5 days. One runner completed the event 47 minutes after the cut off.

Before arrival at the race start, each runner was required to have a medical examination and certification of fitness by his local medical practitioner. Each runner reported to race control on the day before race start. A questionnaire was completed 
Table 1 Male runner characteristics

\begin{tabular}{lll}
\hline & Mean & Range \\
\hline Age & 38 & $23-53$ \\
Running experience (years) & 11 & $1-43$ \\
Years running ultramarathons & 5 & $0-17$ \\
Training distance (km/week) & 227 & $80-500$ \\
\hline
\end{tabular}

which detailed age, previous running history, usual training distance, past and current medical history, recent running related injuries, and medications or supplements currently in use or to be used during the event.

The medical staff of the event consisted of two medical practitioners, one physiotherapist, and one podiatrist. Two of the staff were experienced in the medical care of ultramarathoners and all staff had experience in the diagnosis and management of running injuries. Each runner was encouraged to include a medical practitioner, physiotherapist, or masseur in their crew; however, the most of the crews were only accompanied by a masseur.

The four medical staff collected data during continuous sweeps of the field of runners. Upon arrival at a runner's position, radio contact was made with the runner's crew and inquiry made regarding the condition and medical needs of the runner. Alternatively a medical staff member would speak to the runner while running with him. An injury was recorded only following examination of the runner after a request from the runner or crew, or following a decision by the medical staff member-based on the history of injury - that an examination was appropriate. Each runner was contacted and observed by a member of the medical staff at least twice and up to eight times in each 24 hour period.

\section{Results}

The characteristics of the male runners are detailed in table 1 . Eleven of the males had previously completed this event, two having completed four Sydney to Melbourne runs.

The characteristics of the female runners are detailed in table 2 . One of the females had previously completed this event.

Only three runners indicated medical problems at the time of start of the event. Specifically, minor plantar fasciitis, nonspecific knee pain, and a throat infection under treatment with antibiotics were reported. These problems were observed not to have impaired performance during the event.

The extent of potential self medication during the event was assessed by responses to a question regarding medications or supplements currently used or expected to be used during the event.

Medication usage is presented in table 3 . One participant was using two prescribed

Table 2 Female runner characteristics

\begin{tabular}{lll}
\hline & Mean & Range \\
\hline Age & 43 & $40-46$ \\
Running experience (years) & 10 & $9-13$ \\
Years running ultramarathons & 4 & $1-5$ \\
Training distance (km/week) & 180 & $90-250$ \\
\hline
\end{tabular}

Table 3 Medication/supplement

\begin{tabular}{ll}
\hline Medication/supplement & No of users \\
\hline Multivitamins & 15 \\
Non-steroidal anti-inflammatory drugs & 13 \\
Iron & 7 \\
Vitamin C & 6 \\
Amino acids & 4 \\
Paracetamol & 3 \\
Vitamin B & 2 \\
Ginseng & 2 \\
Calcium & 1 \\
Lecithin & 1 \\
KH3 (Procaine HCL, haematoporphyrin) & 1 \\
\hline
\end{tabular}

medications for hypertension (nifedipine 20 $\mathrm{mg}$ twice daily and prazosin $5 \mathrm{mg}$ three times daily).

INJURIES

Sixty four injuries were seen in 29 runners. Seventy two per cent of runners reported at least one injury. Fifty eight of the injuries $(90 \%)$ involved the lower limb, four $(6 \%)$ were back injuries, and one (1.5\%) each involved the upper limb and chest wall. Sixty three of the injuries were overuse injuries. The number of individual injuries sustained by runners is presented in the figure.

Common injuries and their distribution are presented in table 4 . One case of each of the following was also diagnosed: calf strain, peroneus brevis insertion strain, tibialis posterior insertion strain, thoracic spine pain, lumbar intervertebral joint dysfunction, chest wall pain, tibialis posterior tendinitis, hamstring strain, pes anserine tendinitis, adductor strain, de Quervains tenovaginitis, and anterior talo-fibular ligament sprain.

All participants complained from time to time of excessive muscle tightness, occasional cramps, generalised lower limb soreness, and varying degrees of blistering of the feet. Ankle and hand oedema were also reported. The distribution of injury by region is shown in table 5 .

In view of the requirement for running on the left hand side of the road it was felt that the road camber might play a role in the incidence of injury. When all injuries excluding those to the upper limb and chest wall were considered, 20 occurred on the left side, 14 on the right, 11 bilaterally, and the side of the remainder was not recorded. Analysis of lower limb overuse injuries showed that 19 occurred on the left, 10 on the right, nine bilaterally, and for seven the side was not recorded. Retropatellar pain syndrome (seven cases on the left, three on the right) and tendinitis at the inferior extensor retinaculum (two cases on the left, six on the right, and two unknown) were the only specific injuries that showed a potentially significant lateralisation.

\section{Discussion}

Hutson reported that $60 \%$ of runners participating in a six day event sustained injuries severe enough to impair their performance. ${ }^{4}$ This is in agreement with the present study in which $72 \%$ of the runners were diagnosed with at least one significant injury which would have had a detrimental effect on performance. The top five finishers were found to have 0.6 
Table 4 Common injuries and their distribution

\begin{tabular}{llllll}
\hline Injury & Total & Lefi & Right & Bilateral & Unspecified \\
\hline Retropatellar pain syndrome & 10 & 4 & 0 & 3 & 0 \\
Achilles tendinitis & 5 & 3 & 1 & 0 & 1 \\
Anterior compartment tendinitis & 5 & 1 & 1 & 1 & 1 \\
Extensor digitorum tendinitis & 5 & 0 & 2 & 1 & 1 \\
Medial tibial stress syndrome & 5 & 0 & 1 & 2 & 0 \\
Quadriceps tendinitis & 4 & 1 & 2 & 0 & 1 \\
Anterior compartment pain & 4 & 0 & 0 & 2 & 0 \\
Iliotibial band syndrome & 3 & 2 & 1 & 0 & 0 \\
Quadriceps strain/tear & 3 & 2 & 1 & 0 & 0 \\
Back muscle strain & 2 & 0 & 0 & 1 & 0 \\
Peroneal tendinitis & 2 & 2 & 0 & 0 & 0 \\
Non-specific knee pain & 2 & 0 & 0 & 1 & 0 \\
Greater trochanteric bursitis & 2 & 0 & 0 & 1 & 0 \\
Extensor hallucis longus tendinitis & 2 & 1 & 1 & 0 & 0 \\
\hline
\end{tabular}

injuries per runner, the total finishing group sustained 1.42 injuries per runner and the non-finishers 2.72 per runner. Interestingly, the runner who reported the largest number of injuries (eight) completed the event, albeit in last place.

It would seem unusual that eight runners $(25 \%)$ in an event covering more than $1000 \mathrm{~km}$ would not sustain at least one injury, but this figure is lower than that found by Hutson, ${ }^{4}$ who found that $40 \%$ of his group did not sustain a significant injury. Many runners in these events have experience of minor discomforts and injuries and may tolerate these without complaint, asking for medical assistance only for relatively significant pain or previously unencountered problems.

\section{ANTERIOR ANKLE TENDINITIS}

While the most common single diagnosis made was retropatellar pain syndrome, a greater proportion of the injuries described involved tendinitis of the muscles of the anterior compartment of the lower leg. Twelve injuries ( $19 \%$ of the total) involved these tendons, and in almost every case the major site of inflammation was at the extensor retinaculum at the anterior aspect of the ankle. Hutson reported that $30 \%$ of injuries in his study involved tendinitis of the foot dorsiflexors and in a report involving a $100 \mathrm{~km}$ run Lind makes specific mention of tendinitis of the anterior ankle. ${ }^{7}$

This injury has received scant attention in the medical literature related to running. Only two cases of this group of injuries, specifically

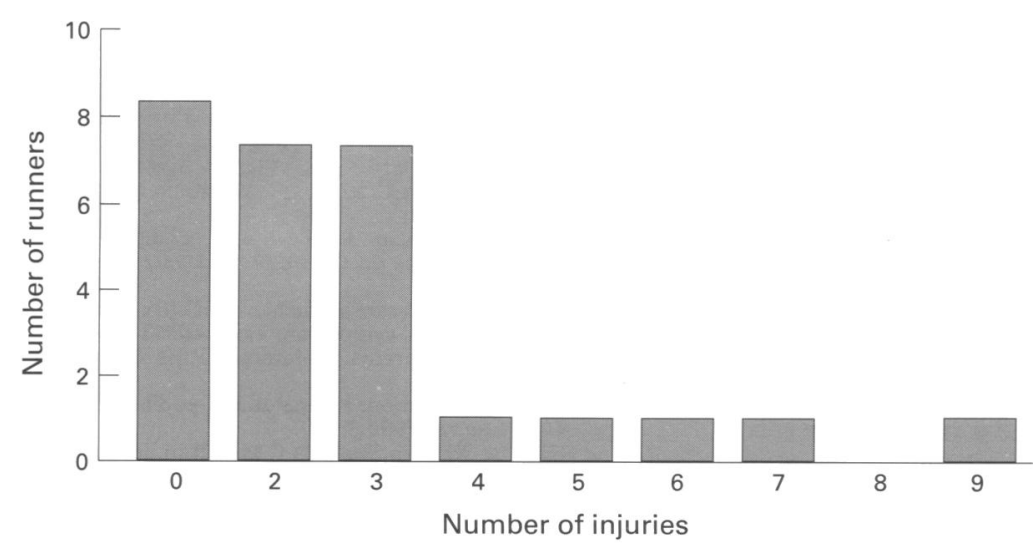

Table 5 Distribution of injury by region

\begin{tabular}{llllll}
\hline Area & Total & Left & Right & Bilateral & Uncertain \\
\hline Knee & 20 & 8 & 2 & 3 & 4 \\
Ankle & 18 & 8 & 5 & 2 & 3 \\
Lower leg & 9 & 1 & 2 & 3 & 0 \\
Upper leg & 7 & 3 & 2 & 1 & 0 \\
Back & 4 & 0 & 0 & 2 & 0 \\
Foot & 4 & 1 & 2 & 0 & 1 \\
Upper limb & 1 & 0 & 0 & 0 & 1 \\
Thorax & 1 & 0 & 1 & 0 & 0 \\
\hline
\end{tabular}

extensor digitorum longus tendinitis, were found in a survey of 1650 patients with 1819 injuries presenting to a sports medicine clinic, ${ }^{1}$ while no mention of these injuries was made in response to a questionnaire survey of 4358 male joggers. ${ }^{8}$

Several studies of triathletes ${ }^{910}$ and ultraendurance triathletes ${ }^{11}$ have determined rates of injury at various anatomical sites and related these to training and other factors, but there are no reports specifically indicating a diagnosis for each injury, therefore no information is available relating to this specific group of injuries in a population whose event may involve durations encountered in shorter ultramarathons. A study of 107 long distance backpackers who, while walking only $18.5 \mathrm{~km}$ per day, continued for a mean of 141 days indicated that tendinitis was one of the more common musculoskeletal problems but again, being a questionnaire study, failed to provide specific information on diagnosis of site of tendon inflammation. ${ }^{12}$

Tendinitis of the "common extensor muscle" is discussed by Marko and Bojanic, ${ }^{13}$ who indicate that the likely cause is excessive dorsal pressure from footwear and that cases resulting from one episode of excessive irritation are rare. Both anterior tibial tendinitis and extensor tendinitis at the ankle are discussed by Subotnick. ${ }^{14}$ Factors considered to be important in the aetiology of anterior tibial tendinitis include excessive pronation of the foot, running on hard surfaces, and overstriding, while overuse, rapid loading of the foot, and tight fitting shoes contribute to extensor tendinitis.

All of the cases in the present study resulted from one episode of unrelenting exercise. The simple number of repetitions of plantar and dorsiflexion of the ankle performed by these tendons under load is the most significant aetiological factor. As the duration of the run increased, many of the runners were seen to adopt a more shuffling type of gait, with a decreased range of dorsi- and plantar flexion at the ankle. This may have led to a relatively small segment of the tendons passing under the extensor retinaculum being continually subjected to friction and irritation. Irritation of the dorsum of the ankle related to excessive pressure of the running shoe may have been a factor, not so much from tight lacing but from diffuse swelling of the ankle and feet found in many participants, much of the ankle swelling being directly related to the peritendinitis itself.

Eccentric overload has been postulated as a major cause of tendinitis. ${ }^{15}$ As decelerators of the foot and ankle the involved tendons clearly 
sustain excessive eccentric load which, combined with muscular fatigue and increased rate of loading, leads to tendon inflammation.

Stretching is thought to decrease the risk of musculotendinous injury; however, stretching of the anterior compartment, limited in part by range of ankle plantar flexion, is relatively difficult and this may predispose to injury.

Ultramarathon running of the distance involved in the Sydney to Melbourne run contains an intrinsic training error. While one runner trained $500 \mathrm{~km} /$ week for this event, the mean distance trained was less than one quarter of the distance of the event. Musculoskeletal tissues are therefore likely to incur damage, as training for all running events usually requires training runs close to or at the distance of the proposed event. This is clearly impossible in this type of event.

Tendinitis/peritendinitis of those structures passing deep to the extensor retinaculum at the ankle, tibialis anterior, extensor digitorum longus, and extensor hallucis longus is an injury only commonly encountered in ultramarathon running. While it may be encountered in other ultraendurance sports this has not been documented. No information is available relating to causative factors specifically related to the ultramarathon, but further research may redress this deficit. Clearly marked overuse and excessive eccentric loading are important in the aetiology of this condition.

\section{OTHER INJURIES}

Patellofemoral or retropatellar pain syndrome accounted for $15.6 \%$ of injuries in the current study, more than double the figure found by Hutson ${ }^{4}$ but less than the $24.3 \%$ noted by Clement et al ${ }^{1}$ in shorter distance runners. This disparity may be explained by the fact that in the six day track race the direction of running was changed every 12 hours in a deliberate attempt to reduce injury. In this study runners ran only on the left hand side of the road. Patellar tendinitis was not documented in the Sydney to Melbourne study but constituted $19 \%$ of injuries in Hutson's study. ${ }^{4}$ Achilles tendinitis was the equal second most frequent diagnosis in both studies.

In previous studies involving runners in general the knee is the region most frequently injured $^{1}$ or is found to be equal in injury rate with the tibial region (including Achilles tendon) and foot (including ankle). ${ }^{8}$ In the present study injury to the knee area was the most frequent, followed closely by the ankle, including Achilles tendon, all of the overuse ankle injuries being tendinitis. In Hutson's study ${ }^{4}$ injuries to the ankle region, including Achilles tendon, were almost twice as frequent as injuries to the knee.

Lower limb injuries were more common on the left, this tendency being more apparent if only overuse injuries of the lower limb were studied. This trend was most marked for retropatellar pain syndrome and the reverse was found for extensor tendinitis at the anterior aspect of the ankle. The finding of left predominance is in agreement with previous research in which injuries were documented in 115 runners involved in an 18-20 month training programme running up to $80 \mathrm{~km} /$ week. ${ }^{16} \mathrm{In}$ this study 93 lower limb injuries occurred on the left and 69 on the right. The authors hypothesised that the road camber led to an unequal load on the legs. Road camber is rarely discussed in surveys of running injuries but has been mentioned as an occasional aetiological factor. ${ }^{1}$

There are few data on the biomechanics of running on cambers, but the effects on knee kinematics have been addressed by Gehlsen et al. ${ }^{17}$ In this study the uphill leg flexionextension range of motion mean values were consistently less than the downhill leg values and there was less internal rotation on the uphill leg in the pronated foot position than on the downhill leg in the supinated foot position. It is difficult to correlate these findings with those of lateralisation of injury in the ultramarathon; however, a significant difference between legs in the varus-valgus displacements in both swing and support phases may lead to sufficient malalignment of the femoro-tibial angle to contribute to a difference in retropatellar pain between sides.

\section{UPPER LIMB INJURY}

The injury to the upper limb, de Quervain's tenosynovitis, was interesting in that it was an overuse injury caused by the runner holding a cassette player in one hand for long periods during the run. The same mechanism led to a case of lateral epicondylitis in the 1989 Sydney to Melbourne run.

CONCLUSION

As the popularity of the marathon wanes, endurance athletes are seeking greater challenges, as exemplified by the current interest in ultraendurance triathlons. Less popular but potentially more stressful to the lower limbs are running ultramarathons. This study attempts to alert sports medicine personnel to the musculoskeletal problems that may be encountered during such an event. It has also highlighted a problem relatively specific to the ultramarathon, that of tendinitis under or adjacent to the extensor retinaculum at the ankle"ultramarathoner's ankle".

1 Clement DB, Taunton JE, Smart GW, McNicol KL. A survey of overuse running injuries. Physician Sportsmed $1981 ; 9: 47-58$

2 Nicholl JP, Williams BT. Injuries sustained by runners during a popular marathon. Br f Sports. Med 1983;17:10-15.

Kretsch A, Duras P, Grogan R, Allen F. Australian Sports Medicine Federation 1980 Big M Marathon Study. Sports Health 1986;4:21-5.

4 Hutson MA. Medical implications of ultramarathon running: observations on a six day track race. $B r F$ Sports Med 1984;18:44-5.

5 Rodriguez A. Medical care at the New York Six day Run. In: Laird RH, ed. Ross symposium on medical coverage of endurance athletic events. Columbus: Ross Laboratories, 1988:14-15.

6 Hawes B. Ultramarathon: a general and podiatric perspective. Aust Podiatrist 1990;3:7-9.

7 Lind RH. The Western States 100 Mile Run. In: Laird RH, ed. Ross symposium on medical coverage of endurance athletic events. Columbus: Ross Laboratories, 1988:26-35.

8 Marti B, Vader JP, Minder CE, Abelin T. On the epidemiology of running injuries. The 1984 Bern Grand-Prix study. Am $\mathcal{F}$ Sports Med 1988;16:285-93. 
9 Williams MM, Hawley JA, Black R, Freke $M$, Simms, K Injuries among competitive triathletes. $N Z \mathcal{F}$ Sports Med Injuries amoni:

10 Collins K, Wagner M, Peterson K, Storey M. Overuse injuries in triathletes. A study of the 1986 Seafair Triathlon. Am $\mathcal{F}$ Sports Med 1989;17:675-80

11 O'Toole ML, Hiller DB, Smith RA, Sisk TD. Overuse injuries in ultraendurance triathletes. Am $\mathfrak{f}$ Sports Med $1989 ; 17: 514-8$.

12 Puretz SL. Health problems of the long-distance backpacker. Aust f Sci Med Sport 1992;24:55-9.

13 Marko P, Bojanic I. Overuse injuries of the musculoskeletal system. Boca Raton: CRC Press, 1993:261.
14 Subotnick SI. The ankle. In: Subotnick SI, ed. Sports medicine of the lower extremity. New York: Churchill Livingstone, 1989:275-6.

15 Curwin S, Stanish WD. Tendinitis: its aetiology and treatment. Lexington: Collamore Press, 1984:25-8.

16 Bovens AMP, Janssen GME, Vermeer HGW, Hoeberigs JH, Janssen MPE, Verstappen FTJ. Occurrence of running injuries in adults following a supervised training program. Int F Sports Med 1989.10(suppl 3):S186-90.

17 Gehlsen GM, Stewart LB, Van Nelson C, Bratz JS. Knee kinematics: the effects of running on cambers. Med $S c i$ Sports Exerc 1989;21:463-6.

\section{SPORTING MISCELLANY \\ Nosejob!}

The proliferation of external nasal dilators in sport has been meteoric over the past 18 months. Initially developed for the noninvasive management of snoring, these small adhesive devices have been adopted by sportsmen worldwide since their first appearance, when worn by the Springboks in the Rugby World Cup in 1995.

The recent television coverage of EURO 96 demonstrated widespread use in international football. Many players feel that these devices help their breathing and hence their performance, but I have been unable to uncover any research providing objective physiological evidence. The psychology of the professional footballer, however, is such that any factor which apparently results in improved personal performance will be faithfully adopted until his next poor performance, when it will be summarily discarded.

For performance to be enhanced by the use of these devices it would be necessary to show a significant increase in oxygen delivery to muscle. We are already familiar with the physiology of pulmonary function, alveolar ventilation, and blood gas transfer in professional footballers. Reilly demonstrated that vital capacity in English professional players averaged 5.8 (SD 0.18) litres as compared with $5.15(0.06)$ litres predicted from height and age nomograms. Similarly, lung power as measured by $\mathrm{FEV}_{1}$ was significantly higher at $4.95(0.16)$ litres $\mathrm{s}^{-1}$ as opposed to $4.3(0.04)$ litres $\mathrm{s}^{-1}$. Maximum minute ventilation would presumably be the variable most influenced by the use of external nasal dilators, and values vary widely among professional footballers, ranging from $108.3(16.19)$ litres $\mathrm{min}^{-1}$ by Williams and colleagues ${ }^{2}$ to 149 (12.4) litres $\mathrm{min}^{-1}$ in 30 Dutch professional players. ${ }^{3}$ It is likely, however, that the only index of pulmonary function which would limit performance in a trained athlete is respiratory muscle fatigue. This would only be produced during sustained maximal performance to exhaustion, which does not occur in football matches.

Given the physiology of pulmonary ventilation in exercise it is difficult to see how improving any minor contribution from nasal airflow will significantly improve maximal minute ventilation.

What then are the advantages to the professional footballer?

There is no doubt that wearing these devices gives a sensation of increased airflow when voluntarily breathing through the nose (does any nasal breathing occur during intensive exercise?). The psychological effect of apparently increased nasal airflow may help when the end of a hard game seems to be a lifetime away!

Although these devices were freely available for use in both training and matches to the England Youth Squad during their recent European Championship Campaign, only a few players tried them once. The room-mate of one player with a significant snoring problem derived great benefit, however, when his colleague wore the device at night!

ANDREW R DENT, Team Physician Stoke City FC \& England Under 18 Football Team

1 Reilly T. What research tells the coach about soccer. Washington DC: AAHPERD.

2 Verstappen F, Bovens F. Interval testing with football players at a laboratory. Science and Football 1989;2:15-16.

3 Williams C, Reid RM, Couttes R. Observations on the aerobic power of university rugby players and professional soccer players. Br $\mathcal{J}$ Sports Med 1973;7:390-1. See also: The encyclopaedia of sports medicine ed Shepherd RI As. The P-O. Oxford: Blackwell Scientific Publications, RJ, Astrat 